\title{
LETTER
}

\section{Dependence of the threshold for perturbation field generated $m / n=2 / 1$ tearing modes on the plasma fluid rotation}

\author{
H.R. Koslowski ${ }^{1}$, Y. Liang ${ }^{1}$, A. Krämer-Flecken ${ }^{1}$, \\ K. Löwenbrück ${ }^{1}$, M. von Hellermann ${ }^{2}$, E. Westerhof ${ }^{2}$, \\ R. C. Wolf ${ }^{1}$, O. Zimmermann ${ }^{1}$ and the TEXTOR team \\ ${ }^{1}$ Forschungszentrum Jülich GmbH, Association EURATOM-FZ Jülich, Institut für \\ Plasmaphysik, Trilateral Euregio Cluster, D-52425 Jülich, Germany \\ ${ }^{2}$ FOM Institute for Plasma Physics Rijnhuizen, Association EURATOM-FOM, Trilateral \\ Euregio Cluster, PO Box 1207, 3430 BE Nieuwegein, The Netherlands
}

Received 1 March 2006, accepted for publication 12 June 2006

Published 25 July 2006

Online at stacks.iop.org/NF/46/L1

\begin{abstract}
The dynamic ergodic divertor (DED) on the TEXTOR tokamak allows for the creation of static and rotating helical magnetic perturbation fields. In the $3 / 1$ configuration the strong $m / n=2 / 1$ sideband excites a locked $2 / 1$ tearing mode above a critical perturbation field strength. The mode onset threshold depends strongly on the plasma fluid rotation with respect to the mode. Rotation in plasma current direction destabilizes the mode in a certain range of rotation frequencies, whereas counter-rotation has a stabilizing influence. The threshold shows a minimum when the frequency of the external perturbation equals the MHD frequency of the mode.
\end{abstract}

PACS numbers: 52.30.Cv, 52.35.Py, 52.55.Tn

\begin{abstract}
Non-axisymmetric error fields are a concern for the next generation of tokamak devices, e.g. ITER. These error fields arise from imperfections of the construction and alignment of the magnetic field coils. The error fields can induce locked tearing modes with low poloidal and toroidal mode numbers, $m$ and $n$. The basic mechanisms for the excitation of tearing modes by non-axisymmetric field components work via toroidal rotation braking and resonant drive when the error field mode numbers match major MHD modes. The most deleterious tearing mode is the $m / n=2 / 1$ mode which grows to large island widths and causes a considerable loss of confinement. The plasma discharge may end up in a disruption of the plasma current when this mode slows down and locks to the wall. Experiments on present day tokamaks (COMPASS-D [1], DIII-D [2], and JET data [3,4]) showed that the relative amplitude of a radial error field normalized to the toroidal magnetic field as low as $B_{r} / B_{\phi}=5 \times 10^{-5}$ is sufficient to excite the $2 / 1$ mode [5]. This is of the same order as the anticipated intrinsic error field resulting from construction and alignment tolerances. As a consequence error field correction systems capable of correcting intrinsic errors have to be provided.
\end{abstract}

Most of the experiments on error field thresholds were done in Ohmic or L-mode plasmas. DIII-D experiments at high beta close to the no-wall beta limit revealed an increasing susceptibility for error field generated mode onset [2]. JET results obtained in lower heating power and mainly L-mode plasmas showed a stabilizing influence of neutral beam heating which was attributed to the effect of rotation because ICRH heating even at higher powers (and H-mode access) had only a small influence on the mode threshold [3]. Despite the investigation of error field thresholds using static fields, rotating magnetic perturbations have been applied on the DITE tokamak in order to feedback stabilize disruption precursor modes [6] and on JFT-2M it has been shown that rotating perturbation fields can transfer toroidal momentum to the plasma [7].

The TEXTOR tokamak is equipped with the dynamic ergodic divertor (DED) [8]. This device consists of a set of 16 helical coils (plus two correction coils to compensate for imperfections at the feeder regions of the coils) mounted on the high-field side of the torus inside the vacuum vessel. The coils can be connected to produce perturbation fields with the fundamental mode numbers $m / n=12 / 4,6 / 2$, and $3 / 1$. 
The coils can be supplied with dc current yielding a static perturbation field or with ac currents resulting in a rotating field. The frequency of the rotating perturbation field can be either low $(\approx 50 \mathrm{~Hz}$ or high frequency in the range 1 to $10 \mathrm{kHz}$. These frequencies are of the order of the electron diamagnetic drift frequency for standard TEXTOR discharge conditions. The maximum coil current depends on the configuration and frequency and can be up to $15 \mathrm{kA}$. In the $m / n=3 / 1$ configuration the current can amount up to $3.75 \mathrm{kA}$ per coil, but since four adjacent coils are connected in parallel the sum of the coil currents is again $15 \mathrm{kA}$.

The experiments reported here were all performed in the $3 / 1$ configuration of the DED using dc and $1 \mathrm{kHz}$ ac. The $3 / 1$ configuration of the DED produces a quite strong $m / n=2 / 1$ sideband perturbation which can excite a $2 / 1$ tearing mode in the plasma. The tearing mode is created phase locked to the DED perturbation field, i.e. it is locked in the tokamak frame (zero frequency) when the DED is operated statically (dc), or it rotates with the DED frequency in dynamic operation (ac). The width of the associated magnetic island in the plasma has been detected as a flattening in the soft $\mathrm{x}$-ray emission profile to be about $8 \mathrm{~cm}$, i.e. $17 \%$ of the plasma minor radius [9].

Mode number analysis based on the phase comparison of toroidally or poloidally arranged magnetic pick-up coils yields the poloidal mode number $m=2$ and the toroidal mode number $n=1$. No mode is present before the application of the DED perturbation field, i.e. the mode has been excited as a consequence of the deliberately applied perturbation field. The process of mode excitation is very reproducible and allows for the investigation of the dependence of the mode excitation threshold, the so-called mode penetration process [10,11], on various plasma parameters.

Time traces of a typical discharge set up to study the $m / n=2 / 1$ mode excitation are shown in figure 1 . The signals from top to bottom show the plasma current, the lineaveraged electron density, the central electron temperature, the DED current, the angular frequency of toroidal rotation measured by charge exchange recombination spectroscopy in the plasma centre (full curve) and at the outboard side close to the location of the $q=2$ rational surface (where positive values indicate plasma rotation in co-current direction), local electron densities measured at the high field side close to the $q=2$ radius, the local electron temperature at the $q=2$ radius and the island width of the $m / n=2 / 1$ tearing mode, measured by a soft x-ray camera. The toroidal magnetic field was $2.25 \mathrm{~T}$. The plasma current was chosen to be $300 \mathrm{kA}$, corresponding to a edge safety factor of $q_{a}=4.5$. This rather high value has been chosen because at lower edge safety factors the excitation of the large and locked mode leads to disruption of the plasma. For the discharge parameters shown in figure 1 safe and stable operation without disruption was achievable.

When the slowly ramped up DED current exceeded a certain value plasma braking occurred, as can be seen from the time trace of the central rotation. Slightly delayed electron temperature at $R=2.05 \mathrm{~m}$ dropped and the electron density at $R=1.57 \mathrm{~m}$ as well as the line-averaged density show a drop. The fast recovery of the drop in electron density is due to the action of the density feedback controller. The simultaneous increase in the electron density at $R=1.44 \mathrm{~m}$ indicates the flattening of the density profile due to the enhanced transport across the generated island.

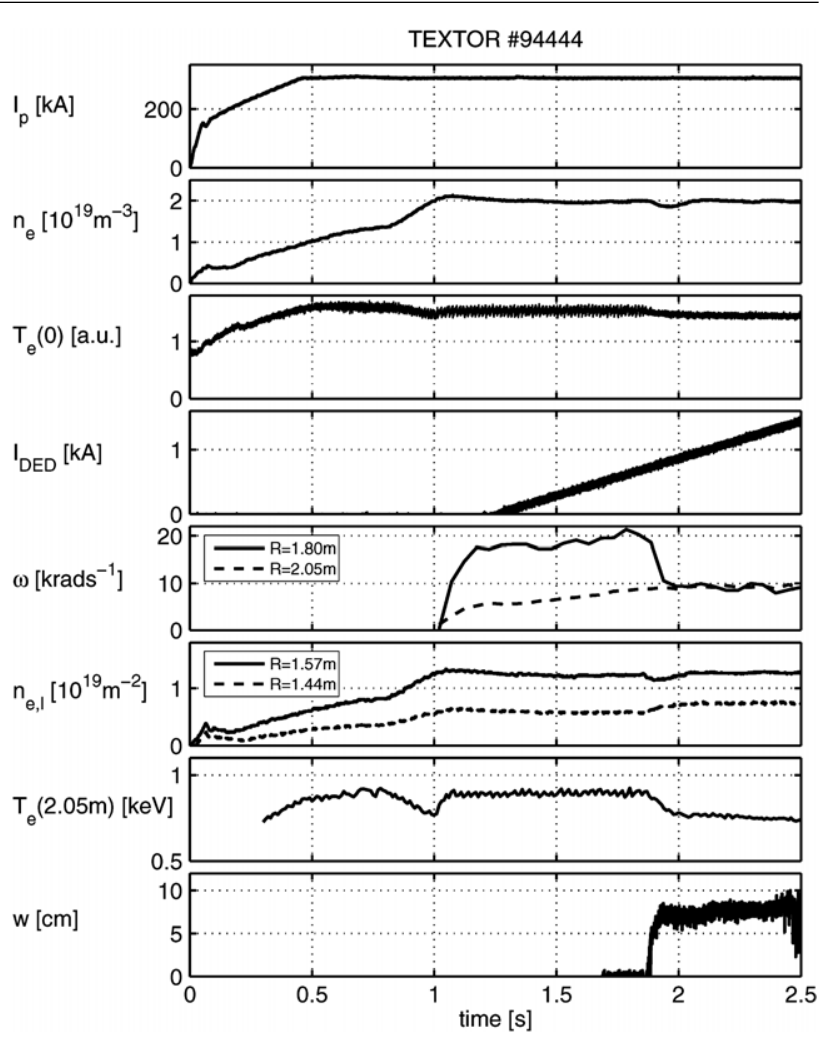

Figure 1. Time traces for a typical discharge to probe the onset threshold for $m / n=2 / 1$ mode excitation. The signals from top to bottom are the plasma current, the line-averaged electron density, the central electron temperature, the DED current, the angular frequency of toroidal rotation measured by charge exchange recombination spectroscopy in the plasma centre (full curve) and at the outboard side close to the location of the $q=2$ rational surface (positive values indicate plasma rotation in co-current direction), local electron densities measured at the high field side close to the $q=2$ radius, the local electron temperature at the $q=2$ radius and the island width of the $m / n=2 / 1$ tearing mode, measured by a soft $\mathrm{x}$-ray camera.

The TEXTOR tokamak is equipped with two neutral beam injectors (NBI), one injecting in the direction of the plasma current (co-NBI), the other one in the opposite direction (cntr-NBI). The injected power of each injector is up to $1.5 \mathrm{MW}$ and can be precisely controlled by adjustable apertures in the beam line, the so called V-targets, which are water cooled calorimeters installed to measure the beam power [12]. Using both NBI and adjusting the V-targets accordingly allows us to scan the beam power and hence the momentum input to the plasma from full co-injection to full counter-injection.

In addition, TEXTOR has an ion cyclotron resonance heating (ICRH) system. Two antennas and generators are installed; each one can deliver up to $2 \mathrm{MW}$ to the plasma. The combination of NBI and ICRH allows to discriminate the influence of plasma pressure (beta) and rotation on the mode onset threshold. This is shown in figure 2 where the DED current threshold for excitation of the 2/1 tearing mode is plotted versus the ICRH power. Two scans have been performed: (i) one scan where various amounts of ICR heating were applied, i.e. the plasma beta increased with power (circles), and (ii) a scan where the sum of ICRH and NBI heating powers was kept constant (triangles). The ICRH 


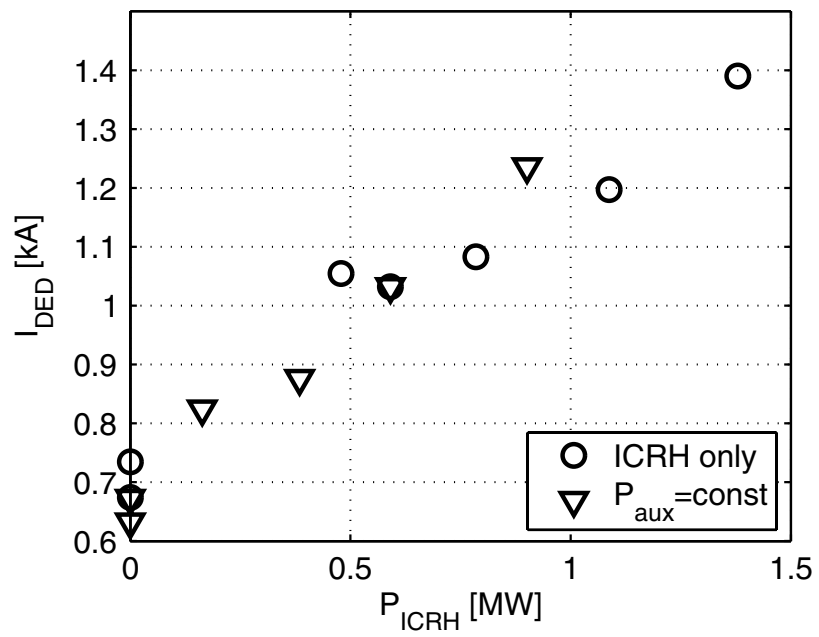

Figure 2. Two scans of the ICRH heating power. The first one $(O)$ shows the influence of beta on the mode excitation threshold. In the second scan $(\triangle)$ beta was kept constant by replacing ICRH power with co-NBI, keeping the total auxiliary heating power constant $(900 \mathrm{~kW})$

only scan shows an increase in mode threshold with heating power or beta. The normalized beta, $\beta_{N}=\beta_{t} /\left(I_{p} /\left(a B_{\phi}\right)\right)$, rises from 0.36 (for $P_{\mathrm{ICRH}}=0$ ) to 0.62 at maximum ICRH power (1.4 MW). The increase of threshold current by a factor of two indicates roughly a linear dependence of the mode excitation threshold with beta in the range of low beta values, similar to the finding in [2]. The second scan shows a very similar behaviour, although the reduction in ICR heating power was always compensated by an increase in co-NBI injection power, keeping the total heating power (and hence beta) constant. Based on a pure beta dependence one would expect the triangles to align horizontally around a value of $1.2 \mathrm{kA}$ coil current, corresponding to the total auxiliary heating power of $900 \mathrm{~kW}$. The decrease with decreasing ICRH power shows that increasing NBI injection in co-current direction is destabilizing.

Using both NBIs allows us to measure the influence of rotation at constant beta. This is shown in figure 3. Here the normalized beta, $\beta_{N}=\beta_{t} /\left(I_{p} /\left(a B_{t}\right)\right)$, remains constant for various mixtures of co- and counter-injection (at constant total power), and plasma temperature and density at the $q=2$ surface are unchanged as well. No ICRH is applied in these discharges.

The dependence of the mode threshold on the plasma rotation has been studied in detail for static and rotating perturbation fields. The results for dc operation of the DED are summarized in figure 4, where the threshold current for two different plasma densities is plotted as a function of the NBI fraction, defined by $f_{\mathrm{NBI}}=\left(P_{\mathrm{co}}-P_{\mathrm{cntr}}\right) /\left(P_{\mathrm{co}}+P_{\mathrm{cntr}}\right)$. The NBI fraction is a simple measure of the momentum input to the plasma. The curves are drawn to guide the eye. Both curves show the same behaviour: Co-NBI, i.e. rotation in co-current direction, has a destabilizing influence on the mode onset threshold. Counter-NBI or counter-rotation is always stabilizing, and co-rotation stabilizes above a certain value. The curves are asymmetric with respect to co and counter rotation. The slope in the direction of counter rotation is smaller than in the direction of co-rotation. Here, a small

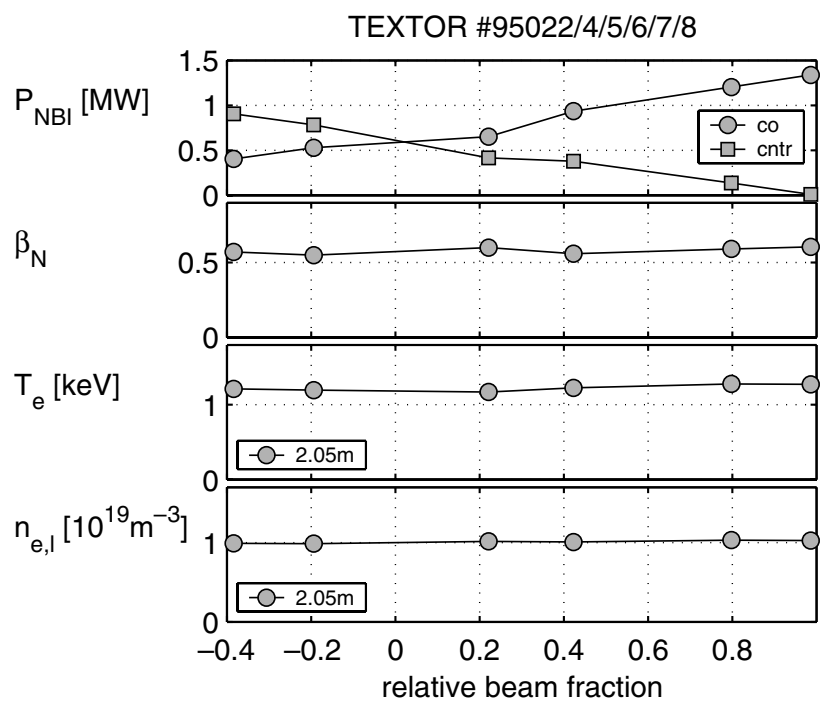

Figure 3. Shown are (from top to bottom): neutral beam heating power of the co- and counter-beams, normalized beta, electron temperature at the $q=2$ surface and electron density at the $q=2$ surface versus the relative beam fraction. The NBI fraction is defined as $\left(P_{\mathrm{co}}-P_{\mathrm{cntr}}\right) / P_{\mathrm{NBI}}$. The plasma rotation changes from counter- to co-current direction, according to the momentum input by neutral heating.

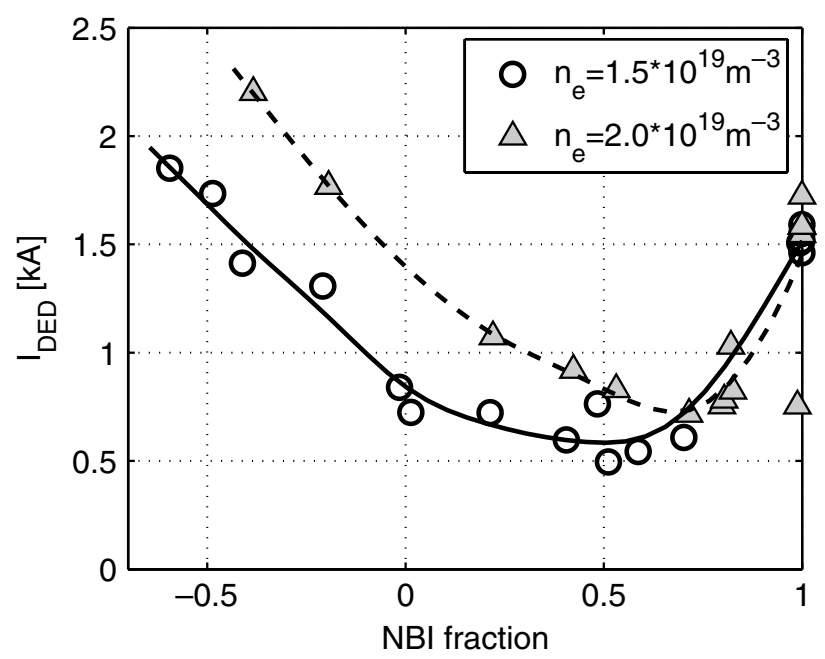

Figure 4. Mode onset threshold versus beam heating composition for two different plasma densities and dc operation of the DED.

change in rotation leads to a large change in mode excitation threshold. The destabilizing influence of lower levels of co-NBI is in contradiction to results from JET [13], where NBI (in co-direction) has been found to be generally stabilizing. A quantitative analysis of the influence of plasma rotation on the field penetration threshold based on JET data yields $B_{\text {pen }} / B \propto \omega^{0.5}[14]$.

The minimum in the dataset at lower density is shifted to smaller co-injection power, i.e. it is located at smaller values of the toroidal plasma rotation (in co-current direction).

A qualitatively similar result was obtained when the dynamic operation of the DED at $1 \mathrm{kHz}$ in counter-current direction was applied. Figure 5 shows the measured threshold currents as a function of the neutral beam composition. 
The current in the DED coils has been rescaled to yield the same effective perturbation field as in the dc case. The minimum value at $n_{e}=1.5 \times 10^{19} \mathrm{~m}^{-3}$ is the same as in the static case (figure 4). Although only two points at the higher density are available, the similar value for the threshold current suggests that the minimum at this density is somewhere between the two points and is shifted to higher co-rotation, similar to the dc case.

For both ac measurements the direction of toroidal rotation of the perturbation field was chosen to be in the counter-current direction. Using a field rotation with $1 \mathrm{kHz}$ in co-current direction did not excite the $2 / 1$ tearing mode, even at the maximum possible coil current.

The comparison of the dc and $1 \mathrm{kHz}$ ac data at the same densities shows that the minimum in the mode penetration threshold is shifted towards lower co-rotation velocities (less co-momentum input from the neutral heating beams) in the ac measurements. The analysis of charge exchange data shows that the minima for the measurements at $n_{e}=1.5 \times 10^{19} \mathrm{~m}^{-3}$ are at toroidal rotation frequencies of $1.7 \mathrm{kHz}$ and $0.7 \mathrm{kHz}$, respectively, i.e. the shift is equal to the frequency of the external perturbation field.

The observed dependence on the toroidal plasma (fluid) rotation can be explained as follows. The plasma rotation needs to be stopped in order to allow the external perturbation field to penetrate. The plasma rotation is generally stabilizing because of the viscosity, counteracting the braking induced by the external electromagnetic torque. This effect is independent of the direction of rotation and has been recently calculated by a quasi-linear MHD model [15]. A second effect is due to the frequency match between the external perturbation field and the MHD frequency of the mode, given by the sum of diamagnetic drift frequency and plasma rotation frequency. The electromagnetic torque exerted on the plasma shows a resonant behaviour and is maximum when the frequencies of mode and external perturbation match. In order to achieve frequency matching and allow mode penetration the toroidal plasma velocity has to be broken (or accelerated) by the electromagnetic torque due to the applied error field [10].

The minimum of the curves corresponds to the resonant condition $f_{\mathrm{DED}}=f_{\mathrm{MHD}}=f_{\phi}-f^{*}$, where $f_{\phi}$ denotes the toroidal plasma rotation frequency and $f^{*}$ the magnitude of the electron diamagnetic drift frequency. The electron diamagnetic frequencies (calculated according to [16]) and the toroidal rotation frequencies for the minima of the curves in figures 4 and 5 are summarized in table 1 . The difference between $f_{\phi}$ and $f^{*}$ is, within the error bars, equal to the frequency of the external perturbation field, $f_{\mathrm{DED}}$. The minus sign reflects the fact that on TEXTOR with the toroidal magnetic field in the opposite direction with respect to the plasma current, $B_{\phi} \uparrow \downarrow I_{p}$, the diamagnetic drift of the mode is in counter-current direction. The resonance condition requires a toroidal plasma rotation in co-current direction. The ac data need less rotation, because the perturbation field rotates in the counter-direction. Reversing the DED field rotation in the co-current direction results in a larger co-plasma rotation to match the frequencies. The required co-rotation cannot be obtained with only one co-beam injector, and the viscosity is even more stabilizing. Therefore no mode excitation has been observed for this rotation direction of the DED field.

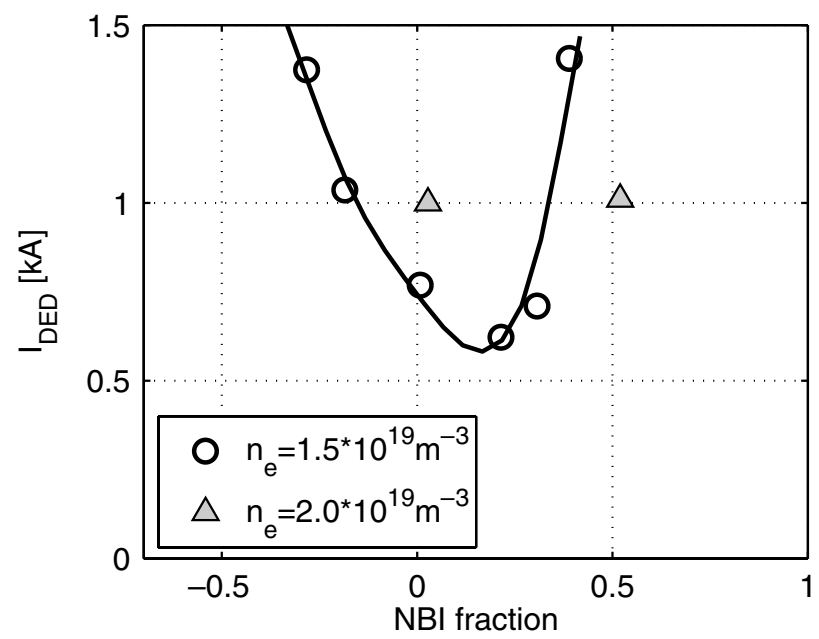

Figure 5. Same as figure 4 but using a counter-rotating perturbation field with a frequency of $1 \mathrm{kHz}$.

Table 1. Rotation frequency of the DED perturbation field, toroidal plasma rotation frequency and electron diamagnetic frequency for the minima of the curves in figures 4 and 5 .

\begin{tabular}{llll}
\hline$n_{e}\left[10^{19} \mathrm{~m}^{-3}\right]$ & $f_{\mathrm{DED}}[\mathrm{kHz}]$ & $f_{\phi}[\mathrm{kHz}]$ & $f^{*}[\mathrm{kHz}]$ \\
\hline 2.0 & 0 & 1.3 & 1.6 \\
1.5 & 0 & 1.7 & 1.6 \\
1.5 & -1 & 0.7 & 1.6 \\
\hline
\end{tabular}

The strong dependence of the error field penetration threshold on the difference between MHD frequency of the mode and the frequency of the external field offers a straightforward explanation of the apparently strong beta dependence seen in figure 2. The mode frequency strongly depends on beta and rises from $f=1 \mathrm{kHz}$ at $500 \mathrm{~kW}$ ICRH power to $2.1 \mathrm{kHz}$ at $1400 \mathrm{~kW}$ heating power, i.e. $\left|f^{*}\right|$ increases with higher beta and thus electron temperature at fixed density. When comparing the strong influence of ICRH heating on the mode excitation threshold on TEXTOR to the rather weak effect found on JET [3], it seems likely that the dominant contribution is due to the increase in $f^{*}$ which results in an increase in frequency mismatch and requires a stronger perturbation field to brake the differential rotation between the external field and the MHD frequency of the mode.

The same influence of beta argument is probably true for NBI heating, but here the momentum input and spin-up of the plasma rotation reduces the mode frequency and lowers the mode onset threshold. A stabilizing influence of increasing plasma pressure cannot be neglected totally but seems to be small in the present experiment. Future experiments on TEXTOR where the total beam power is gradually increased and the balance between co- and counter-injection adjusted to keep the difference $f_{\phi}-f^{*}$ constant may serve to answer this question.

The reverse $B$ campaign in 2003 on the JET tokamak allowed for the investigation of the error field mode threshold for co- and counter-rotation. The JET data show the minimum in the penetration threshold at zero beam power, i.e. no momentum input [17]. This discrepancy is not understood at present. A similar difference was found in the minimum of the sawtooth period as function of the plasma rotation, 
which on TEXTOR [18] and JET [19] occur when the $n=1$ precursor frequency is zero. TEXTOR shows the minimum at co-momentum injection, whereas at JET it is at counter beam injection.

In summary, the recently installed DED on the TEXTOR tokamak allows the investigation of the mode penetration process due to a strong $m / n=2 / 1$ sideband perturbation. In combination with the flexible neutral beam system, which allows a precise control of the balance between co- and counterinjection, and hence the momentum input to the plasma, the dependence of the mode threshold on the toroidal plasma rotation could be studied. The mode penetration threshold has a minimum at a certain toroidal plasma rotation frequency when the MHD frequency of the mode matches the frequency of the applied perturbation field. This has been directly confirmed by the shift of the minimum in the excitation threshold curves using dynamic, rotating perturbation fields.

\section{References}

[1] Hender T.C. et al 1992 Nucl. Fusion 322091

[2] La Haye R.J. et al 1992 Nucl. Fusion 322119

[3] Buttery R.J. et al 1999 Nucl. Fusion 391827
[4] Buttery R.J. et al 2000 Nucl. Fusion 40807

[5] ITER Physics Expert Group on Disruptions, Plasma Control and MHD, ITER Physics Base Editors, ITER EDA 1999 Nucl. Fusion 392251

[6] Morris A.W. et al 1990 Phys. Rev. Lett. 641254

[7] Oasa K. et al 1994 Proc. 15th Int. Conf. on Plasma Physics and Controlled Nuclear Fusion Research (Seville, Spain, 1994) (IAEA) vol 2 p 279

[8] Finken K.H. (ed) 1997 Special issue: dynamic ergodic divertor Fusion Eng. Des. 37335

[9] Liang Y. et al 2004 Proc. 31st EPS Conf. on Plasma Physics (London, UK, 2004) (ECA) vol 28G P-1.126

[10] Fitzpatrick R. 1993 Nucl. Fusion 331049

[11] Fitzpatrick R. 1998 Phys. Plasmas 53325

[12] Uhlemann R. and Ongena J. 1999 Fusion Technol. 3542

[13] Fishpool G.M. and Haynes P.S. 1994 Nucl. Fusion 34109

[14] Lazzaro E. et al 2002 Phys. Plasmas 93906

[15] Kikuchi Y. et al 2006 Plasma Phys. Control. Fusion 48169

[16] Chen Y.J. et al 1990 Research using small tokamaks Proc. Tech. Comm. Meeting (Arlington, 1990) (Vienna: IAEA) $\mathrm{p} 41$

[17] Buttery R.J. et al 2003 Error field scalings and sideband effects-an update Post APS-DPP 2003 Error Field Workshop (Albuquerque, New Mexico, USA)

[18] Koslowski H.R. 2005 Fusion Sci. Technol. 47260

[19] Nave M.F. et al 2006 Phys. Plasmas 13014503 